\title{
Gastrointestinal Bleeding Risk Factors in Patients with Type 2 Diabetes Mellitus
}

\author{
Savas Volkan Kisioglu ${ }^{1}$, Ihsan Habiboglu², Yasemin Emur Gunay ${ }^{3}$, Serdar Durak ${ }^{4}$, Serdar Karakullukcu ${ }^{5}$ and \\ Sami Fidan ${ }^{4}$ \\ ${ }^{1}$ Department of Endocrinology, Istinye University, Yildizli Medical Park Hospital, Trabzon, Turkey \\ ${ }^{2}$ Department of Gastroenterology, Sivas Numune Hospital, Sivas, Turkey \\ ${ }^{3}$ Department of Endocrinology, Karadeniz Technical University, Trabzon, Turkey \\ ${ }^{4}$ Department of Gastroenterology, Karadeniz Technical University, Trabzon, Turkey \\ ${ }^{5}$ Department of Public Health, Bayburt Community Health Centre, Bayburt, Turkey
}

\begin{abstract}
Objective: To investigate the factors leading to the development of gastrointestinal bleeding (GIB) by comparing patients with diabetes mellitus Type 2 (T2DM) with dyspeptic complaints without GIB; and patients with T2DM who had GIB, regardless of the presence of helicobacter pylori.

Study Design: Analytical study.

Place and Duration of Study: Department of Endocrinology and Gastroenterology, Faculty of Medicine, Karadeniz Technical University, from January 2018 to June 2019.

Methodology: The patients were divided into GIB and dyspepsia groups. After the identification of patients in both groups, demographic characteristics, drugs, comorbidities, presence of diabetic macro- and micro-vascular complications, and endoscopic findings were examined retrospectively for each patient.

Results: There were 106 patients, with 53 patients in each group. Mean age was significantly higher in the GIB group compared to the dyspepsia group $(p<0.001)$. Body mass index (BMI) was significantly lower in the GIB group $(p<0.001)$. Frequency of congestive heart failure (CHF), chronic kidney disease (CKD), and cerebrovascular disease (CVD), heart valve disease, and cardiac arrhythmia was significantly higher in GIB group ( $p<0.05$ for all). No significant correlation was found between acetylsalicylic acid (ASA) use and GIB $(p=0.103)$. The use of nonsteroidal anti-inflammatory drugs (NSAID), novel oral anticoagulants (NOAC), and clopidogrel was significantly higher in the GIB group ( $p=0.032, p=0.031$, and $p=0.032$, respectively). Proton pump inhibitor (PPI) use was significantly higher in the dyspepsia group $(p=0.002)$.

Conclusion: Age, and poly medications were associated with increased frequency of GIB. The use of ASA, when not administered with other agents that may induce GIB, does not increase the risk of developing GIB in obese T2DM patients younger than 65 years of age, who have increased HbAlc levels.
\end{abstract}

Key Words: Type 2 diabetes mellitus, Dyspepsia, Gastrointestinal bleeding, Acetylsalicylic acid, Risk factors, Obesity, Medication.

How to cite this article: Kisioglu SV, Habiboglu I, Gunay YE, Durak S, Karakullukcu S, Fidan \&. Gastrointestinal Bleeding Risk Factors in Patients with Type 2 Diabetes Mellitus. J Coll Physicians Surg Pak 2022; 32(01):15-19.

\section{INTRODUCTION}

Cardiovascular diseases are the most common cause of mortality in patients with diabetes mellitus type 2 (T2DM); and the American Diabetes Association (ADA) guidelines recommend the initiation of low dose acetylsalicylic acid (ASA) in diabetic patients with cardiovascular risk factors after evaluating the benefit-harm ratio in detail. ${ }^{1}$

Correspondence to: Dr. Savas Volkan Kisioglu, Department of Endocrinology, Istinye University, Yildizli Medical Park Hospital, Trabzon, Turkey

E-mail: volkankisioglu@yahoo.com

Received: July 29, 2021; Revised: October 28, 2021;

Accepted: November 09, 2021

DOI: https://doi.org/10.29271/jcpsp.2022.01.15
In addition, patients with T2DM have an increased risk of gastrointestinal bleeding (GIB) following the use of ASA compared to non-diabetic patients. ${ }^{2}$

Administration of helicobacter pylori ( $\mathrm{Hp}$ ) eradication to reduce the risk of GIB in patients scheduled for ASA administration remains controversial. ${ }^{3}$ Despite varying among societies, there are numerous limiting factors for $\mathrm{Hp}$ eradication, including high rate of positivity, difficult eradication, and the risk of reinfection. ${ }^{4-6}$ For this reason, as a more practical solution, proton pump inhibitors (PPI) can be used for GIB prophylaxis in patients scheduled for ASA administration. ${ }^{7}$

In this study, the aim was to investigate the factors leading to the development of GIB by comparing patients with T2DM who had dyspeptic complaints but had no GIB; and patients with T2DM who had GIB, regardless of the presence of $\mathrm{Hp}$. 


\section{METHODOLOGY}

The study included patients with T2DM who underwent endoscopy at Karadeniz Technical University, Faculty of Medicine, Gastroenterology Unit, between January 2018 and June 2019. Patients with T2DM who were hospitalised due to major GIB (hematemesis or melena), which was confirmed by hospital personnel and caused by a peptic ulcer lesion as determined in an endoscopic procedure, were included in GIB group. Cases with any other cause of bleeding, in-hospital bleeding, and prior Hp eradication were excluded. For dyspepsia group, patients selected among patients who, with T2DM scheduled for ASA administration and had dyspeptic complaints, and who had undergone gastroscopy. Patients with a history of GIB and Hp eradication were excluded. Medicines used within 72 hours before gastroscopy were recorded for each patient. ASA doses below $300 \mathrm{mg}$ were accepted as low dose. The GIB group was formed from the intersection of ICD codes for gastrointestinal hemorrhage (K92) and T2DM (E11).

Data were analysed using SPSS for Windows version 23.0 (Armonk, NY: IBM Corp.). Categorical variables were expressed as frequencies ( $n$ ) and percentages (\%) and continuous variables were expressed as median and minimum-maximum values mean and standard deviation for normally distributed, and median with IQR for non-normally distributed. Categorical variables were compared using Chi-square test and Fisher's Exact test. Independent continuous variables were compared using Student t-test when normal distribution condition was met, and Mann-Whitney U-test when not. Independent continuous variables were compared using Student t-test when they show a normal distribution and Mann-Whitney U-test when they did not show a normal distribution. A p-value of $<0.05$ was considered significant.

\section{RESULTS}

Initially, there were 53 patients; in the T2DM group and 56 patients in the GIB group. The three youngest patients in the dyspepsia group were excluded from the study and the remaining 53 patients who were closest to the GIB group in terms of age, were included in the study.

Table Ipresents the demographiccharacteristicsand comorbidities of the patients. Mean age was above 60 years in both groups, while it was significantly higher in the GIB group compared to the Dyspepsia group $(p<0.001)$. Body mass index (BMI) was significantly lower in the GIB group $(p<0.001)$. Frequency of congestive heart failure (CHF), chronic kidney disease (CKD), and cerebrovascular disease (CVD), heart valve disease, and cardiac arrhythmia was significantly higher in GIB group ( $p<0.05$ forall).

Table II presents the drugs used by the patients. No significant correlation was found between ASA use and GIB $(p=0.103)$. In the GIB group, 11 out of 23 patients using ASA were receiving second agents that could cause a GIB. Isolated use of ASA was noted in $12(23 \%)$ patients in the GIB group and in 14 (26\%) patients in the dyspepsia group. In the comparison of patients using ASA only, no significant correlation was found between ASA use and GIB ( $p=0.821)$. After excluding patients using PPI from patients who used ASA only, there were $9(17 \%)$ patients in the GIB group and 7 (13\%) patients in the dyspepsia group. In these patients, no significant correlation was found between ASA and GIB $(p=0.776)$. The number of patients using anticoagulants and/or antithrombotics and/or nonsteroidal anti-inflammatory drugs (NSAID) was significantly higher in the GIB group $(p<0.001)$. Moreover, the use of NSAID, novel oral anticoagulants (NOAC), and clopidogrel was significantly higher in the GIB group ( $p=0.032, p=0.031$, and $p=0.032$, respectively). In the GIB group, 4 out of 15 patients using multiple agents that could cause GIB were using PPI; whereas, no patient in the dyspepsia group was using multiple drugs that could cause GIB. No significant relationship was found between metformin and insulin use and GIB ( $p=0.181$ and $p=0.231$, respectively). PPI use was significantly higher in the dyspepsia group $(p=0.002)$. In the dyspepsia group, $9(69 \%)$ out of 13 patients with CAD were using PPI; while in the GIB group, only 7 (32\%) out of 22 patients with CAD were using PPI. In the dyspepsia group, 30 patients were using PPI, while 12 of them were using an agent that could cause GIB. In the GIB group, 13 patients were using PPI and 43 patients were using an agent that could cause GIB.

Table III presents the laboratory parameters of the patients. HbAlc values were similar in both groups $(p=0.073)$.

\section{DISCUSSION}

The results indicated that the use of low-dose ASA alone does not increase the prevalence of GIB. It was also revealed that age and additional comorbidities are the primary factors causing increased prevalence of GIB.

Fujimoto et al. analysed the clinical data of 13 million patients aged 20-74 years and found that upper gastrointestinal system bleeding was most common in males and in individuals aged $60-74$ years. ${ }^{8}$ Similarly, in this study, advanced age was associated with a significant increase in the development of GIB. Of note, $87 \%(n=46)$ of the patients with GIB were aged over 60 years and $70 \%(n=37)$ of them were aged over 65 years. Moreover, $58.5 \%$ of the patients with GIB were males, which was at the limit of significance when compared to the dyspepsia group $(p=0.052)$.

Chi et al. evaluated NSAID-related GIB in hospitalised patients aged over 60 years and reported that history of cardiovascular and cerebrovascular disease, DM, antiplatelet drugs, upper abdominal discomfort, anorexia and NSAID used for 0.5 to 3 months were associated with GIB. ${ }^{9}$ Similarly, in this study, the prevalence of GIB was significantly higher in patients with lower BMI. Additionally, as seen in Table I, the risk of GIB increased as the prevalence of comorbidities increased, except for chronic obstructive pulmonary disease (COPD) and CAD. Nevertheless, the limited number of patients with COPD in both groups may not provide a clear evaluation regarding this issue. On the other hand, no significant difference was found between the two groups with regard to the number of 
patients with $C A D$, although the number of patients with $C A D$ was higher in the GIB group. This finding could be due to higher rate of PPI use in the dyspepsia group (Table II). The use of both antithrombotics and PPI have increased over recent years. ${ }^{8}$ The protective efficacy of PPI against GIB is known; ${ }^{7,10,11}$ whereas, many reservations have been noted in the literature regarding their long-term use. ${ }^{12}$ In addition, a newly published meta-analysis reported that long-term use of PPI increases the risk of developing gastric cancer. ${ }^{13}$

Table I: Demographic and clinical characteristics.

\begin{tabular}{|c|c|c|c|c|}
\hline \multirow{2}{*}{\multicolumn{2}{|c|}{ Variables }} & \multicolumn{2}{|l|}{ Groups } & \multirow{2}{*}{$p$} \\
\hline & & GIB $(n=53)$ & Dyspepsia $(n=53)$ & \\
\hline \multicolumn{2}{|l|}{ Age $(\text { year) })^{\dagger}$} & $70(24-89)$ & $62(35-78)$ & $<0.001^{*}$ \\
\hline \multirow{2}{*}{ Sex } & Male & $31(58.5)$ & $21(39.6)$ & \multirow{2}{*}{$0.052^{* *}$} \\
\hline & Female & $22(41.5)$ & $32(60.4)$ & \\
\hline \multicolumn{2}{|l|}{ Weight $(\mathrm{kg})^{\dagger}$} & $75(50-120)$ & $85(65-117)$ & $0.001^{*}$ \\
\hline \multicolumn{2}{|l|}{ BMI $\left(\mathrm{kg} / \mathrm{m}^{2}\right)^{\dagger}$} & $27.5(18.4-42.5)$ & $32.7(23.0-46.3)$ & $<0.001^{*}$ \\
\hline \multicolumn{2}{|c|}{ Duration of diabetes (years) ${ }^{\dagger}$} & $8(1-28)$ & $10(2-34)$ & $0.077^{*}$ \\
\hline \multirow{2}{*}{ Smoking $^{\ddagger}$} & Yes & $4(7.5)$ & $3(5.7)$ & \multirow{2}{*}{$1.000^{* * *}$} \\
\hline & No & $49(92.5)$ & $50(94.3)$ & \\
\hline \multirow{2}{*}{ Hypertension $^{\ddagger}$} & Yes & $38(71.7)$ & $37(69.8)$ & \multirow{2}{*}{$1.000^{* *}$} \\
\hline & No & $15(28.3)$ & $16(30.2)$ & \\
\hline \multirow{2}{*}{$C A D^{\ddagger}$} & Yes & $22(41.5)$ & $13(24.5)$ & \multirow{2}{*}{$0.098^{* *}$} \\
\hline & No & $31(58.5)$ & $40(75.5)$ & \\
\hline \multirow{2}{*}{$\mathrm{CHF}^{\ddagger}$} & Yes & $13(24.5)$ & $0(0.0)$ & \multirow{2}{*}{$<0.001^{* *}$} \\
\hline & No & $40(75.5)$ & $53(100.0)$ & \\
\hline \multirow{2}{*}{$C K D^{\ddagger}$} & Yes & $16(30.2)$ & $6(11.3)$ & \multirow{2}{*}{$0.031^{* *}$} \\
\hline & No & $37(69.8)$ & $47(88.7)$ & \\
\hline \multirow{2}{*}{$\mathrm{CVD}^{\ddagger}$} & Yes & $10(18.9)$ & $2(3.8)$ & \multirow{2}{*}{$0.032^{* *}$} \\
\hline & No & $43(81.1)$ & $51(96.2)$ & \\
\hline \multirow{2}{*}{$\mathrm{COPD}^{\ddagger}$} & Yes & $4(7.5)$ & $3(5.7)$ & \multirow{2}{*}{$1.000^{* * * *}$} \\
\hline & No & $49(92.5)$ & $50(94.3)$ & \\
\hline \multirow{2}{*}{ Heart valve disease ${ }^{\ddagger}$} & Yes & $6(11.3)$ & $0(0.0)$ & \multirow{2}{*}{$0.027^{* * *}$} \\
\hline & No & $47(88.7)$ & $53(100.0)$ & \\
\hline \multirow{2}{*}{ Arrhythmia ${ }^{\ddagger}$} & Yes & $11(20.8)$ & $1(1.9)$ & \multirow{2}{*}{$0.006^{* *}$} \\
\hline & No & $42(79.2)$ & $52(98.1)$ & \\
\hline
\end{tabular}

Table II: Medicines used by the patients.

\begin{tabular}{|c|c|c|c|c|}
\hline \multirow{2}{*}{ Variables } & & \multicolumn{2}{|l|}{ Groups } & \multirow{2}{*}{$p$} \\
\hline & & $\mathrm{GIB}^{\ddagger}(n=53)$ & Dyspepsia $^{\ddagger}(n=53)$ & \\
\hline \multirow{2}{*}{ Aspirin } & Yes & $23(43.4)$ & $14(26.4)$ & \multirow{2}{*}{$0.103^{*}$} \\
\hline & No & $30(56.6)$ & $39(73.6)$ & \\
\hline \multirow{2}{*}{ Warfarin } & Yes & $7(13.2)$ & $1(1.9)$ & \multirow{2}{*}{$0.060^{* *}$} \\
\hline & No & $46(86.8)$ & $52(98.1)$ & \\
\hline \multirow{2}{*}{ LMWH } & Yes & $1(1.9)$ & $0(0.0)$ & \multirow{2}{*}{$1.000^{* *}$} \\
\hline & No & $52(98.1)$ & $53(100.0)$ & \\
\hline \multirow{2}{*}{ Clopidogrel } & Yes & $10(18.9)$ & $2(3.8)$ & \multirow{2}{*}{$0.032^{*}$} \\
\hline & No & $43(81.1)$ & $51(96.2)$ & \\
\hline \multirow{2}{*}{ NOAC } & Yes & $8(15.1)$ & $1(1.9)$ & \multirow{2}{*}{$0.031^{* *}$} \\
\hline & No & $45(84.9) I$ & $52(98.1)$ & \\
\hline \multirow{2}{*}{ NSAID } & Yes & $10(18.9)$ & $2(3.8)$ & \multirow{2}{*}{$0.032^{*}$} \\
\hline & No & $43(81.1)$ & $51(96.2)$ & \\
\hline \multirow{2}{*}{ Multiple anticoagulant or antithrombotic } & Yes & $15(28.3)$ & $0(0.0)$ & \multirow{2}{*}{$<0.001^{*}$} \\
\hline & No & $38(71.7)$ & $53(100.0)$ & \\
\hline \multirow{2}{*}{ Any anticoagulant or antithrombotic } & Yes & $43(81.1)$ & $19(35.8)$ & \multirow{2}{*}{$<0.001^{*}$} \\
\hline & No & $10(18.9)$ & $34(64.2)$ & \\
\hline \multirow{2}{*}{ Steroids } & Yes & $0(0.0)$ & $4(7.5)$ & \multirow{2}{*}{$0.118^{* *}$} \\
\hline & No & $53(100.0)$ & $49(92.5)$ & \\
\hline \multirow{2}{*}{ Steroids + NSAID } & Yes & $0(0.0)$ & $6(11.3)$ & \multirow{2}{*}{$0.027^{* *}$} \\
\hline & No & $53(100.0)$ & $47(88.7)$ & \\
\hline \multirow{2}{*}{ Metformin } & Yes & $36(67.9)$ & $43(81.1)$ & \multirow{2}{*}{$0.181^{*}$} \\
\hline & No & $17(32.1)$ & $10(18.9)$ & \\
\hline \multirow{2}{*}{ Insulin } & Yes & $36(67.9)$ & $29(54.7)$ & \multirow{2}{*}{$0.231^{*}$} \\
\hline & No & $17(32.1)$ & $24(45.3)$ & \\
\hline \multirow{2}{*}{ PPI } & Yes & $13(24.5)$ & $30(56.6)$ & \multirow{2}{*}{$0.002^{*}$} \\
\hline & No & $40(75.5)$ & $23(43.4)$ & \\
\hline
\end{tabular}


Table III: Laboratory parameters.

\begin{tabular}{|c|c|c|c|}
\hline \multirow{2}{*}{ Variable } & \multicolumn{2}{|l|}{ Groups } & \multirow{2}{*}{$p$} \\
\hline & GIB $(n=53)$ & Dyspepsia $(n=53)$ & \\
\hline $\operatorname{HbAlc}(\%)^{*}$ & $6.7(4.8-10.2)$ & $7.0(5.4-15.4)$ & $0.073^{* *}$ \\
\hline Creatinine $^{*}$ & $1.1(0.6-7.5)$ & $0.8(0.4-4.0)$ & $<0.001^{* *}$ \\
\hline Albumin ${ }^{*}$ & $3.3(1.9-4.5)$ & $4.1(1.9-4.8)$ & $<0.001^{* *}$ \\
\hline $\mathrm{BUN}^{*}$ & $47(14-173)$ & $16(9-90)$ & $<0.001^{* *}$ \\
\hline $\mathrm{ALT}^{*}$ & $14(3-72)$ & $15(3-93)$ & $0.229^{* *}$ \\
\hline $\mathrm{LDH}^{*}$ & $202(92-900)$ & $186(125-514)$ & $0.099^{* *}$ \\
\hline WBC $^{*}$ & $10430(1178-58370)$ & $7600(3720-13800)$ & $<0.001^{* *}$ \\
\hline $\mathrm{PNL}^{*}$ & $7190(2140-46570)$ & $4280(1780-9900)$ & $<0.001^{* *}$ \\
\hline Lymphocyte $^{*}$ & $1610(470-6610)$ & $2280(590-4140)$ & $0.007^{* *}$ \\
\hline $\mathrm{Hgb}^{\dagger}$ & $9.4 \pm 2.8$ & $12.6 \pm 1.9$ & $<0.001^{*}$ \\
\hline $\mathrm{PLT}^{\dagger}$ & $206484.9 \pm 79902.1$ & $246792.5 \pm 58636.2$ & $0.004^{*}$ \\
\hline $\mathrm{MPV}^{¥}$ & $10.1(7.0-13.6)$ & 10.5 (7.9-13.7) & $0.013^{* *}$ \\
\hline $\mathrm{CRP}^{*}$ & $0.7(0.0-8.6)$ & $0.4(0.1-7.1)$ & $0.042^{* *}$ \\
\hline
\end{tabular}

In this study, the analysis of four patients that were using prophylactic PPI while using multiple agents that could cause GIB indicated that PPI treatment did not show adequate efficacy in preventing GIB, while using multiple agents that could cause GIB.

In this study, the rate of ASA use was higher in the GIB group, though no significant difference was established $(p=0.103)$. Moreover, after excluding agents that could cause GIB other than ASA, it was observed that the use of ASA did not lead to a significant increase in the risk of GIB, regardless of the use of PPI. In ASCEND-P, 15480 patients with T2DM without significant cardiovascular disease were randomised to aspirin $100 \mathrm{mg}$ once daily or placebo; and the results indicated that major bleeding occurred in 314 (4.1\%) patients using aspirin and in 245 (3.2\%) patients using placebo $(p=0.003)$. Additionally, a significant portion $(25 \%)$ of the major bleedings described in the study occurred in the upper GI tract. In the same study, upper GI endoscopy was performed in 195 (2.5\%) patients in the ASA group and in $205(2.6 \%)$ patients in the placebo group due to dyspeptic complaints. ${ }^{14}$ The fact that the number of patients who needed upper Gl endoscopy was similar, could be considered as an indirect indicator that the use of ASA does not cause an increase in dyspeptic symptoms. Another cohort study evaluated 186,425 individuals and suggested that the use of aspirin was associated with the risk of major bleeding in most of the subgroups studied; and that the use of ASA in individuals with DM did not increase the risk of bleeding. ${ }^{15}$ When evaluated in light of the findings, the findings of the authors suggest that the use of ASA increases the risk of GIB. However, this outcome cannot be attributed to the use of ASA alone. Comorbidities and concomitant medications are additional factors leading to the development of GIB. The findings of this study showed that the use of ASA did not cause a serious risk in GIB development, particularly in obese patients, and individuals younger than 65 years of age, and patients with high $\mathrm{HbAlc}$ values.
Although T2DM is a prothrombotic condition, there are reports that T2DM is an independent risk factor for $\mathrm{GIB}^{15,16}$ In this study, HbAlc values were similar between the two groups, which suggests that GIB is independent of the severity of T2DM. This study was limited due to its retrospective data collection and small patient population.

\section{CONCLUSION}

The use of ASA, when not administered with other agents that may induce GIB, does not increase the risk of developing GIB in obese T2DM patients, younger than 65 years of age, who have increased $\mathrm{HbAlc}$ levels. Instead of initiating prophylactic ASA in each patient, it would be wiser to initiate ASA after considering individual characteristics and risk factors of the patients.

\section{ACKNOWLEDGEMENT:}

The authors wish to thank Prof Dr. Orhan Ozgur and Prof. Dr. Halil Onder Ersoz for their outstanding support.

\section{ETHICAL APPROVAL:}

The study protocol was approved by Karadeniz Technical University, Ethics Committee (24237859-491).

\section{PATIENTS' CONSENT:}

Consent forms were taken from the patients before endoscopic procedure.

\section{CONFLICT OF INTEREST:}

The authors declared no conflict of interest.

\section{AUTHORS' CONTRIBUTION:}

SVK: Conception and design of work, analysis and inter-pretation of data, drafting and revision of work.

$\mathrm{IH}$ : Conception and drafting of the work.

YEG: Design and revision of the work.

SD: Conception of work, analysis and interpretation of data revision.

SK: Design of work, interpretation of data, and revision of the work. 
SF: Conception of work, analysis and interpretation of data and revision of work.

All authors gave final approval of the version to be published, agreed to be accountable for all aspects of the work.

\section{REFERENCES}

1. Cardiovascular disease and risk management: Standards of medical care in diabetes - 2021. Diabetes Care 2021; 44(Supplement 1):S125-S150. doi: 10.2337/dc21-S010.

2. Wang $Y$, Wang $W$, Wang $B$, Wang $Y$. The risk of gastrointestinal hemorrhage in low-dose aspirin users with diabetes mellitus: Systematic review and meta-analysis. Gastroenterology Res Pract 2020; 2020:9824615. doi : $10.1155 / 2020 / 9824615$.

3. Fletcher EH, Johnston DE, Fisher CR, Koerner RJ, Newton JL, Gray CS. Systematic review: Helicobacter pylori and the risk of upper gastrointestinal bleeding risk in patients taking aspirin. Aliment Pharmacol Ther 2010; 32(7):831-9. doi: 10.1111/j.1365-2036.2010.04415.x.

4. Zamani M, Ebrahimtabar F, Zamani V, Miller WH, AlizadehNavaei R, Shokri-Shirvani J, Derakhshan MH. Systematic review with meta-analysis: The worldwide prevalence of helicobacter pylori infection. Aliment Pharmacol Ther 2018; 47(7):868-76. doi: 10.1111/apt.14561.

5. Savoldi A, Carrara E, Graham DY, Conti M, Tacconelli E. Prevalence of antibiotic resistance in Helicobacter pylori: A systematic review and meta-analysis in World health organisation regions. Gastroenterol 2018; 155(5):1372-82. doi: 10.1053/j.gastro.2018.07.007.

6. Xie $\mathrm{Y}$, Song $\mathrm{C}$, Cheng $\mathrm{H}$, Xu C, Zhang Z, Wang J, et al. Society of gastroenterology, chinese study group on helicobacter pylori and peptic ulcer. Long-term follow-up of helicobacter pylori reinfection and its risk factors after initial eradication: A large-scale multicentre, prospective open cohort, observational study. Emerg Microbes Infect 2020; 9(1):548-557. dol: 10.1080/22221751.2020.1737579.

7. García Rodríguez LA, Lanas A, Soriano-Gabarró M, Vora P, Cea Soriano L. Effect of proton pump inhibitors on risks of upper and lower gastrointestinal bleeding among users of low-dose aspirin: A population-based observational study. J
Clin Med 2020; 9(4):928. doi: 10.3390/jcm9040928.

8. Fujimoto S, Tsuruoka N, Esaki M, Takamori A, Sakata Y, Shimoda R, Fujimoto K. Decline incidence in upper gastrointestinal bleeding in several recent years: Data of the Japan claims database of 13 million accumulated patients. J Clin Biochem Nutrition 2021; 68(1):95-100. dol: 10.3164/jcbn.20-153.

9. Chi TY, Zhu HM, Zhang M. Risk factors associated with nonsteroidal anti-inflammatory drugs (NSAIDs)-induced gastrointestinal bleeding resulting on people over 60 years old in Beijing. Medicine (Baltimore) 2018; 97(18):0665. doi: 10.1097/MD.0000000000010665.

10. Haastrup, PF, Hansen JM, Søndergaard J, Jarbøl DE. Proton pump inhibitor use among patients at risk of peptic ulcer bleeding: a nationwide register-based study. Scandinavian J Gastroenterol 2021; 56(1):6-12. doi: 10.1080/00365521. 2020.1853220.

11. Matsuda H, Nosaka T, Akazawa Y, Saito Y, Ozaki Y, Takahashi K, NakamotoY. Daily usage of proton pump inhibitors may reduce the severity of critical upper gastrointestinal bleeding in elderly patients. Gastroenterol Res Practice 2020; 2020. doi: $10.1155 / 2020 / 7168621$.

12. Koyyada A. Long-term use of proton pump inhibitors as a risk factor for various adverse manifestations. Therapies 2021; 76(1):13-21. doi: 10.1016/j.therap.2020.06.019.

13. Lin JL, Lin JX, Zheng $\mathrm{C} \mathrm{H}$, Xie JW, Wang JB, Lu J. et al. Longterm proton pump inhibitor use and the incidence of gastric cancer: A systematic review and meta-analysis. J Gastric Surg 2020; 2(1):1-11. doi: 10.36159/jgs.v2i1.17.

14. ASCEND study collaborative group. "Effects of aspirin for primary prevention in persons with diabetes mellitus. New Eng J Med 2018; 379.16:1529-39. dol: 10.1056/NEJMoa 1804988.

15. De Berardis G, Lucisano G, D'Ettorre A, Pellegrini F, Lepore $V$, Tognoni G, Nicolucci, A. Association of aspirin use with major bleeding in patients with and without diabetes. JAMA 2012; 307(21):2286-94. doi: 10.1001/jama.2012.5034.

16. Luo PJ, Lin XH, Lin CC, Luo JC, Hu HY, Ting PH, Hou MC. Risk factors for upper gastrointestinal bleeding among aspirin users: An old issue with new findings from a population-based cohort study. J Formosan Med Assoc 2019; 118(5): 939-44. doi: 10.1016/j.jfma.2018.10.007. 